\title{
Dried blood spot omega-3 and omega-6 long chain polyunsaturated fatty acid levels in 7-9 year old Zimbabwean children: a cross sectional study
}

Grace Mashavave ${ }^{1 *}$, Patience Kuona ${ }^{2}$, Willard Tinago ${ }^{3}$, Babill Stray-Pedersen ${ }^{4}$, Marshall Munjoma ${ }^{5}$ and Cuthbert Musarurwa'

\begin{abstract}
Background: Omega-3 long chain-polyunsaturated fatty acids (LC-PUFAs)-docosahexaenoic acid (DHA), docosapentaenoic acid (DPA) and eicosapentaenoic acid (EPA)- and omega-6 LC-PUFA arachidonic acid (ARA), are essential for optimum physical and mental development in children. Prior to this study, the blood omega-3 LC-PUFA levels were unknown in Zimbabwean children, particularly in those aged 7-9 years, despite the documented benefits of LC-PUFAs. Documentation of the LC-PUFA levels in this age group would help determine whether interventions, such as fortification, are necessary. This study aimed to determine dried whole blood spot omega-3 and omega-6 LC-PUFA levels and LC-PUFA reference intervals among a selected group of Zimbabwean children aged 7-9 years old.

Methods: We conducted a cross sectional study from September 2011 to August 2012 on a cohort of peri-urban, Zimbabwean children aged 7-9 years. The children were born to mothers enrolled at late pregnancy into an HIV prevention program between 2002 and 2004. Dried whole blood spots were sampled on butylated hydroxytoluene antioxidant impregnated filter papers and dried. LC-PUFAs were quantified using gas liquid chromatography. Differences in LC-PUFAs between groups were compared using the Kruskal Wallis test and reference intervals determined using non-parametric statistical methods.

Results: LC-PUFAs levels were determined in 297 Zimbabwean children of whom 170 (57.2\%) were girls. The study determined that LC-PUFAs (wt/wt) ranges were EPA 0.06-0.55 \%, DPA 0.38-1.98 \%, DHA 1.13-3.52 \%, ARA 5.58-14.64 \% and ARA: EPA ratio 15.47-1633.33. Sixteen participants had omega-3 LC-PUFAs levels below the determined reference intervals, while 18 had higher omega-6 LC-PUFAs. The study did not show gender differences in omega-3 and omega-6 LC-PUFAs levels (all $p>0.05$ ). EPA was significantly higher in the 8 year age group compared to those aged 7 and 9 years (median; 0.20 vs 0.17 vs 0.18 , respectively, $p=0.049$ ). ARA: EPA ratio was significantly higher in the 7 year age group compared to those aged 8 and 9 years (median; 64.38 vs 56.43 vs 55.87 respectively, $p=0.014$ ).

Conclusions: In this cohort of children, lower EPA levels and higher ARA: EPA ratios were observed compared to those reported in apparently healthy children elsewhere. The high ARA: EPA ratios might increase the vulnerability of these children to inflammatory pathologies. Identification and incorporation into diet of locally produced foodstuffs rich in omega-3 LC-PUFAs is recommended as well as advocating for dietary supplementation with omega-3 fish oils and algae based oils.
\end{abstract}

Keywords: Omega-3 long chain-polyunsaturated fatty acids, Docosahexaenoic acid, Eicosapentaenoic acid, Docosapentaenoic acid, Arachidonic acid, Dried blood spot, Children, 7-9 year old

\footnotetext{
* Correspondence: gracemashavave@yahoo.com

'Department of Chemical Pathology, College of Health Sciences, University

of Zimbabwe, PO BOX A178, Avondale, Harare, Zimbabwe

Full list of author information is available at the end of the article
} 


\section{Background}

Omega-3 long chain-polyunsaturated fatty acids (LCPUFAs)-docosahexaenoic acid (DHA), eicosapentaenoic acid (EPA) and docosapentaenoic acid (DPA) - are essential for growth, development and general health [1]. Omega-6 LC-PUFA arachidonic acid (ARA) is essential for brain development [2]. DHA is especially critical for optimal brain [2], cognitive [3] and behavior development. EPA is a precursor of anti-inflammatory eicosanoids (prostaglandins (3 series), leukotrienes (5 series) and thromboxanes $\left(\mathrm{TXA}_{3}\right)$ ) [4], and adequate intake of EPA is closely related to positive immunological [5], inflammatory [6] and metabolic [7] outcomes. DPA has been reported to have beneficial effects which include inhibiting platelet aggregation, stimulating endothelial cell migration and regulating gene expression [8]. Though Arachidonic acid (ARA) is a precursor of pro-inflammatory eicosanoids (prostaglandins ( 2 series), leukotrienes (4 series) and thromboxanes( TXA $\left._{2}\right)$ ) [4]; it produces some metabolites that are required for systemic homeostasis [9]. ARA and its metabolite lipoxin $\mathrm{A}_{4}$ have been shown to function as endogenous antidiabetic molecules [10]. ARA together with LC-PUFAs and their anti-inflammatory products: lipoxins, resolvins, protectins and maresins suppress production of proinflammatory eicosanoids, limit inflammation, enhance wound healing, resolve inflammation thus restoring normal cellular, tissue and organ function [10].

Supplementation with omega-3 LC-PUFAs in cardiovascular diseases (CVD) [11], diabetes mellitus [12], hypertension [13], sickle cell anemia [14], inborn errors of metabolism [15, 16], non-alcoholic fatty liver disease [17, 18], attention-defect/hyperactivity disorder [19] autism [20] and asthma [21] has been reported to prevent or alleviate symptoms in children, though other studies have reported conflicting results [22-25].

The above disorders are due to deficiencies in omega3 fatty acids. Deficiencies in omega- 3 fatty acids may result from factors that affect availability of omega-3 LC-PUFAs and influence the metabolism of essential fatty acids (EFA) to LC-PUFAs. These include, imbalances in metabolic pathways [26], genetics [27], imbalances in ARA: EPA ratios [28] and sex hormones [29]. The imbalances in the metabolic pathway may result from linoleic acid (LA) competing with $\alpha$-linolenic acid (ALA) for the endogenous conversion of ALA to the long chain derivatives EPA and DHA and also inhibition of incorporation of DHA and EPA into tissues [26]. Therefore high levels of LA in the diet result in low ALA and low omega-3 LCPUFA levels. This in turn affects the omega-6: omega-3 (ARA: EPA) ratios which are critical in human health outcomes [20,28]. The high levels of LA lead to increased activity of the ARA metabolic pathway [4], which has deleterious effects such as neurological and neurodevelopmental disorders [30]. High concentrations of ARA compete with EPA for incorporation into cell membrane phospholipid leading to high ARA: EPA ratios [28]. The low omega-3 LC-PUFA levels can be due to deficiencies and defects in the $\Delta 6$ or $\Delta 5$ desaturase enzyme [31] or mutations in the fatty acid desaturase (FADS) gene [27]. Protein malnutrition, carnitine and $\alpha$-tocopherol enzyme deficiency as well as excess oxygen free radical production in chronic diseases also affect LC-PUFA availability [32]. Oestrogen and testosterone, have been reported to affect EFA metabolism hence availability of long chain metabolites, leading to higher levels in females compared to males [29]. The conversion of EFA into their long chain metabolites is stimulated by oestrogen and inhibited by testosterone [29].

Despite the reported benefits of omega-3 LC-PUFAs in children, most studies demonstrating the nutritional importance of omega-3 LC-PUFAs in children have been carried out in developed countries [2, 3, 11-13], [15-17, 19, 21] with a limited number of studies on African children [14, 33-35]. In most of sub-Saharan Africa healthcare facilities, omega-3 LC-PUFA levels are not on clinical laboratories test menus because the laboratories lack the expertise and technology to perform the tests [36]. In the past the assessment of omega-3 LC-PUFAs has been hindered by difficult methodology [37] and sample instability [38, 39]. Analysis has since been revolutionized by the use of minimally invasive dried blood spots (DBS) [40], which allow the estimation of fatty acid composition of red blood cells and plasma phospholipids that are more reflective of the nutritional status [41]. To date, only a few studies have developed protocols for testing LC-PUFAs in DBS [37-40, 42], with none being carried out in Africa.

In Southern Africa, a region with high prevalence of childhood infectious diseases [43], the levels of omega-3 LC-PUFAs in children are unknown, except in South Africa where the positive effects of omega-3 LC-PUFA supplementation on cognitive development were reported in children aged between 6 and 11 years [33-35]. There is paucity of data on omega-3 LC-PUFA levels in the rest of African countries, including Zimbabwe, especially in children whose adequate intake of LC-PUFAs should be ensured for cognitive development and other positive health outcomes [44].

Monitoring of fatty acid levels and results interpretation in individual patients or in populations require availability of reference intervals obtained from apparently healthy individuals [45]. Fatty acids reference intervals have been established for glycerophospholipids in German children aged 2 and 6 years, for whole blood in apparently healthy European children aged 3-8 years [46] and in apparently healthy Spanish children who were on a normal diet for their age elsewhere [15] but 
these may not be transferable to a different population. However, scanty studies have been done in low income settings particularly in African children and none in Zimbabwe. At present no reference intervals for LCPUFAs have been established in these settings.

In this study, the levels of omega-3 LC-PUFAs were determined in Zimbabwean children aged between 7 and 9 years using DBS and reference intervals for LC-PUFAs were determined. The LC-PUFAs were compared between groups by gender and by age.

\section{Methods}

\section{Study design and setting}

From September 2011 to August 2012 we conducted a cross sectional study at three peri-urban primary health care clinics around Harare, the capital city of Zimbabwe. Children aged 7 to 9 years born between 2002 and 2004 to a cohort of mothers previously recruited at late pregnancy from a national HIV prevention of mother to child transmission (PMTCT) program [47], were invited to participate in the study. These children were also eligible if they had no major chronic diseases. Children who were not born to the specified cohort, or who were siblings to the original cohort or whose care givers declined to allow them to take part in the study were excluded as well as those who were HIV-infected, those with inflammatory pathologies and other chronic diseases like diabetes mellitus that may influence the fatty acid composition. All the children were tested for HIV infection at birth, at 6 weeks and subsequently after 18 months.

The study protocol and consent forms were approved by the Joint Research Ethics Committee: (JREC/170/12), and the Medical Research Council of Zimbabwe: (MRCZ/B/359). The consent forms were also approved by the Norwegian Research Ethics Committee. Permission to ship participant samples abroad for laboratory analysis was granted by the Research Council of Zimbabwe. Written informed consent to participate in the study and for long term specimen storage and shipping was obtained from parents or legal guardians and written assent was also obtained from all the children.

\section{Blood collection}

After cleaning, warming and punching a fingertip with an automatic lancing device equipped with a sterile lancet, a drop of non-fasting capillary whole blood sample $(\sim 50 \mu \mathrm{l})$ from each participant was spotted onto each of the four pre-defined circles on a Whatman 903 (Lot number 6833909/82) filter paper cards (GE Healthcare, UK), which was impregnated with anti-oxidant (butylated hydroxytoluene (BHT; $50 \mathrm{mg} / 100 \mathrm{ml}$ in ethanol) (Sigma Aldrich Limited, Gillingham Dorset, UK)), for the determination of omega-3 LC-PUFA levels [39].
BHT in Ethanol was prepared at a concentration of $0.05 \%$. Each spot on the Whatman 903 filter paper card to be used for blood collection was impregnated with the BHT in ethanol solution, by fully covering each collection spot with the solution (by adding two drops). The cards were air-dried for an hour and placed in a sealed polyethylene bag overnight. The filter paper cards were treated with the $\mathrm{BHT}$ antioxidant to prevent the polyunsaturated fatty acids losses due to oxidation. The samples were air-dried for $3 \mathrm{~h}$ and stored at $-25^{\circ} \mathrm{C}$ in a zip lock foil paper (Whatman, Maidstone/Banbury UK) with a desiccant (Whatman, Maidstone/Banbury UK) [39].

\section{Preparation of fatty acid methyl esters (FAMEs) in DBS, extraction, purification and detection \\ Fatty acid extraction and detection}

Analysis of PUFAs was carried out at the Nutrition Group Laboratories, Institute of Aquaculture at the University of Stirling, Scotland UK, using a fingertip rapid method that was evaluated by Marangoni et al. [40], and validated by Bell et al. [39]. One DBS circle (per sample), one DBS circle (internal standard), and one DBS circle (control) were cut out from the main DBS filter paper cards using a pair of scissors and forceps and were placed each in a pre-labeled $10 \mathrm{ml}$ screw cap vial which was loaded on the CTC-PAL machine carousel. At the beginning and end of each batch of samples or once a week, a marinol FAME secondary reference material and the Supelco 37 controls were run simultaneously with each run/batch of participants to ensure reproducibility of the known control values. The fatty acids from participants' samples, standards and quality control samples were transesterified to fatty acid methyl esters (FAMEs) using 1.25 M HCL/Methanol and incubated in dry heating block at $70{ }^{\circ} \mathrm{C}$ for $1 \mathrm{~h} \mathrm{[39]}$. This was a modification of the one step direct transmethylation method described by Lepage and Roy [48]. The FAMEs were extracted into iso-hexane (Fisher Scientific, Loughborough, UK) and precipitated with saturated potassium chloride (Fisher Scientific, Loughborough, UK) before purification [39]. Purification was done by passing the extract through a preconditioned solid phase extraction (SPE) cartridge (Clean-Up Extraction Columns, UCT, Bristol, USA) (prewashed with iso-hexane) and eluted with isohexane-diethyl ether $(95: 5 v / v)$ (Fisher Scientific, Loughborough, UK) [39]. The FAME extracts were dried under nitrogen (BOC Gases, Guildford, UK) using a nitrogen evaporator $\left(\mathrm{N}-\mathrm{EVAP}^{\mathrm{in}} 111\right.$, Organomation Associates, Berlin USA) at room temperature, re-dissolved in $0.2 \mathrm{ml}$ iso-hexane and placed in an autosampler vial (Chromacol, Herts, UK) prior to gas liquid chromatography (GLC) (Thermo Fisher Trace, Hertfordshire UK) analysis [39]. The stability of the fatty acid analytes in whole wet blood and in the dried whole blood samples 
at different temperatures (room temperature, 4 and $\left.-20{ }^{\circ} \mathrm{C}\right)$ and at different times periods $((3 \mathrm{~h}$ post drying) and subsequent samples were removed for analysis after $48 \mathrm{~h}, 7$ days, 14 days and 28 days in storage) was investigated by Bell et al. [39].

The GLC was calibrated using duplicate injections of standard mixtures of known composition (Supelco AOCS No. 37 standards containing 14:0 to 22:6n-3). A second standard, a Supelco custom mix containing 14:0, 16:0, 16:In-7, 18:0, 18:In-7, 18:2n-6, 10:0, 10:5n-3, 22:In-9, $22: 5 n-3$ and $22-6 n-3$, was used to check calibration and replication by making three consecutive analyses. FAMEs $(1 \mu \mathrm{l})$ were injected, separated and quantified by GLC using a $60 \mathrm{~m} \times 0.32 \mathrm{~mm} \times 0.25 \mu \mathrm{m}$ film thick capillary column (ZB Wax; Phenomenex, Macclesfield, Cheshire, UK). Hydrogen gas (BOC Gases, Guildford, UK) was used as a carrier gas at a flow rate of $4.0 \mathrm{ml} / \mathrm{min}$ and the temperature program was from 50 to $150{ }^{\circ} \mathrm{C}$ at $40{ }^{\circ} \mathrm{C} / \mathrm{min}$ then to $195{ }^{\circ} \mathrm{C}$ at $2{ }^{\circ} \mathrm{C} / \mathrm{min}$ and finally to $215{ }^{\circ} \mathrm{C}$ at $0.5{ }^{\circ} \mathrm{C} / \mathrm{min}$ [39]. The FAMEs were detected by a flame ionization detector. The FAMEs were compared to wellcharacterized in-house standards as well as commercial FAME mixtures (Supelco ${ }^{\text {tm }} 37$ FAME mix; Sigma Aldrich Limited, Gillingham Dorset, UK). Fatty acids ranging from C14:0 to C22:6 carbons were detected. The fatty acid data was collected from the GLC and processed using the Chromocard software computer package for Windows (version 2.1) (Thermoquest Italia S. p. A., Milan, Italy). The FAMEs results were expressed as percent weight of individual fatty acids in total fatty acids (\% weight/weight $(\% w t / w t))$. Precision and coefficient of variation were done by Bell et al. using the same analytical method, on same instrumentation and in the same establishment as the work published in this manuscript.

The total omega-6 LC-PUFAs measured included; Dihomo-gamma-linolenic acid (DGLA), ARA and Docosapentaenoic acid (DPA) (n-6) whereas the total omega-3 LC-PUFAs included; EPA, DPA (n-3) and DHA. Omega-6 LC-PUFAs were included in the results since they compete with omega-3 LC-PUFAs for the same enzymes for desaturation. The results of EPA, DPA (n-3), DHA, ARA, and the calculated total omega-3 PUFAs, total omega-3 LC-PUFA: total omega LCPUFAs, ARA: EPA, total omega-6 LC-PUFA: total omega-3 LC-PUFA ratios were selected for data analysis.

\section{Statistical analysis}

Participants' characteristics were summarized using percentages for categorical variables and median [interquartile ranges (IQR)] for continuous non-normal variables. Differences in median omega-3 LC-PUFAs by age group and by gender were compared using the Kruskal-Wallis test. To account for multiple comparisons, we applied the Bonferroni correction which lowers (adjust) the $\alpha$ value (type1 error) to account for the number of comparisons being performed simultaneously, thereby avoiding a lot of spurious positives. Nonparametric statistical methods from Reference Value Advisor v1.3 [49] were used to determine the central $95 \%$ reference interval limits, with the lower limit defined as the 2.5 percentile and the upper limit defined as the 97.5 percentile, together with the $90 \%$ confidence intervals (CI) of the distribution of omega-3 LC-PUFAs. For all statistical comparisons $\alpha$ ( $p$ value) was set at 0.05 . Data entry and analysis was conducted using Statistical Package for Social Scientists (SPSS, New York, USA).

\section{Results}

A total of 319 of the available children from the original cohort participated in the study. Of these children, one had a DBS collected on a non BHT treated filter paper and 21 (6.6 \%) were HIV-infected. Those who were HIV infected were all excluded from further analysis. Of the remaining 297, the median age (range) was 9 (7-9) years and $170(57.2 \%)$ were girls (Table 1$)$.

\section{Distribution and comparison of LC-PUFA levels}

Sixteen (5.39 \%) of the children had EPA, DPAn-3, DHA levels that were below the determined reference intervals and $18(6.06 \%)$ had ARA levels, ARA: EPA and total omega-6 PUFA: Total omega-3 PUFA ratios that were above the determined reference intervals. The LC-PUFAs (\% $w t / w t)$ ranges were as follows: EPA 0.06-0.55 \%, DPA 0.38-1.98 \%, DHA $1.13-3.52 \%$, ARA $5.58-14.64 \%$ and ARA: EPA ratio 15.47-1633.33 (Table 2).

The median LC-PUFA levels between boys and girls were not significantly different $(p>0.05)$.

EPA levels were significantly elevated in the 8 year age group compared to those aged 7 and 9 years ( 0.17 vs 0.20 vs 0.18 , respectively, $p=0.049)$. The median ARA: EPA ratio was significantly elevated in the 7 year age group compared to the other age groups $(p=0.014)$ (Table 2). Distribution of median ARA: EPA ratio was significantly different between age groups after being corrected using the Bonferoni test (Fig. 1).

Table 1 Age and gender of the children

\begin{tabular}{ll}
\hline Variable & Frequency $n=297$ \\
\hline Gender of the children & $127(42.8 \%)$ \\
Boys & $170(57.2 \%)$ \\
Girls & \\
Children age group (years) & $21(7.1 \%)$ \\
7 & $93(31.3 \%)$ \\
8 & $183(61.6 \%)$ \\
9 & $p$-values calculated using Bonferroni test. Statistically Significance $(p<0.05)$ \\
(2-tailed) &
\end{tabular}


Table 2 Distribution of median (IQR) LC-PUFA levels (\%wt/wt) by gender and age group of 297 Zimbabwean children

\begin{tabular}{|c|c|c|c|c|c|c|c|c|c|}
\hline & \multirow[t]{2}{*}{ Variables } & \multicolumn{4}{|c|}{ LC-PUFAs (\% wt/wt) } & \multirow[b]{2}{*}{$\begin{array}{l}\text { Total Omega-3 } \\
\text { LC-PUFA }\end{array}$} & \multirow[b]{2}{*}{$\begin{array}{l}\text { \% Omega LC-PUFA: } \\
\text { Total LC-PUFA }\end{array}$} & \multirow[b]{2}{*}{ ARA: EPA } & \multirow[b]{2}{*}{$\begin{array}{l}\text { Total n-6 PUFA: } \\
\text { Total n-3 PUFA }\end{array}$} \\
\hline & & EPA & DPA & DHA & ARA & & & & \\
\hline & Median (IQR) $(n=297)$ & $0.18(0.15-0.23)$ & $0.79(0.70-0.89)$ & $2.14(1.87-2.42)$ & $10.62(9.77-11.38)$ & $3.13(2.83-3.49)$ & $18.42(17.10-19.92)$ & $57.47(44.72-72.24)$ & $10.82(9.83-11.79)$ \\
\hline & Mean (SD) $(n=297)$ & $0.20(0.071)$ & $0.81(0.17)$ & $2.15(0.40)$ & $10.56(1.26)$ & $3.55(0.53)$ & $18.11(2.27)$ & $61.31(23.64)$ & $10.91(1.62)$ \\
\hline & Range All $(n=297)$ & $0.06-0.55$ & $0.38-0.198$ & $1.13-3.52$ & $5.58-14.64$ & $1.73-5.95$ & $13.34-28.11$ & $15.47-163-33$ & $5.94-16.03$ \\
\hline \multirow{2}{*}{$\begin{array}{l}\text { Children's gender } \\
\text { median IQR) }\end{array}$} & Boys $(n=127)$ & $0.19(0.15-0.23)$ & $0.81(0.72-0.91)$ & $2.11(1.91-2.46)$ & $10.55(9.80-11.32)$ & $3.25(2.62-3.42)$ & 18.38 (17.32-19.94) & $57.16(45.24-71.82)$ & $10.71(9.78-11.68)$ \\
\hline & Girls $(n=170)$ & $0.18(0.15-0.24)$ & $0.78(0.70-0.89)$ & $2.15(1.84-2.36)$ & $10.67(9.73-11.46)$ & $3.10(2.82-3.47)$ & 18.44 (16.94-19.89) & $57.72(44.09-72.91)$ & $10.95(9.88-11.97)$ \\
\hline \multirow{3}{*}{$\begin{array}{l}\text { Children's Age group } \\
\text { median (IQR) }\end{array}$} & (7) $(n=21)$ & $0.17(0.13-0.18)$ & $0.78(0.74-0.86)$ & $2.27(2.03-2.48)$ & $10.89(10.68-11.75)$ & $3.18(2.92-3.53)$ & 18.20 (16.69-19.52) & 64.38 (59.71-91.04) & $10.60(9.93-11.89)$ \\
\hline & (8) $(n=93)$ & $0.20(0.16-0.24)$ & $0.81(0.70-0.93)$ & $2.16(1.93-2.51)$ & $10.63(9.61-11.39)$ & $3.18(2.87-3.57)$ & $18.64(17.35-20.18)$ & 56.43 (41.39-72.02) & $10.55(9.68-11.45)$ \\
\hline & (9) $(n=183)$ & $0.18(0.15-0.23)$ & $0.79(0.70-0.89)$ & $2.10(1.83-2.35)$ & $10.48(9.76-11.35)$ & $3.09(2.80-3.40)$ & 18.34 (16.94-19.88) & 55.87 (44.90-70.64) & $10.94(9.87-12.01)$ \\
\hline$p$-values & & $P=0.049^{\mathrm{a}}$ & & & & & & $P=.014^{\mathrm{a}}$ & \\
\hline
\end{tabular}

$p$-values calculated using Kruskal Wallis Test Asterisks: Significance tests: ${ }^{\text {a }}$ Statistically Significance $(p<0.05)(2$-tailed)

$\begin{array}{ll}\text { Key to Tables 2-3 } & \text { Eicosapentaenoic acid; } \\ \text { EPA (20:5n-3): } & \text { Docosapentaenoic acid (n-3); } \\ \text { DPA (22:5n-3): } & \text { Docosahexaenoic acid } \\ \text { DHA (22:6n-3): } & \text { Arachidonic acid } \\ \text { ARA (20:4n-6) } & \text { (EPA + DPA + DHA) } \\ \text { Total Omega-3 PUFA } & \text { (DGLA + ARA + AA + DPA (n-6)) } \\ \text { Total Omega-6 PUFA } & \text { [(EPA + DPA + DHA)]: [(DGLA + ARA + AA + DPA (n-6)) + (EPA + DPA + DHA)] } \\ \text { \% Omgea-3 LC-PUFA: Total LC-PUFA } & \text { (DGLA + ARA + AA + DPA (n-6)): (EPA + DPA + DHA) } \\ \text { Total n-6 PUFA: Total n-3 PUFA } & \text { Arachidonic acid: Eicosapentaenoic acid } \\ \text { ARA: EPA } & \text { Dihomo-gamma-linolenic acid } \\ \text { DGLA (20:3n-6) } & \text { Adrenic acid } \\ \text { AA (22:4n-6) } & \text { Docosapentaenoic acid (n-6) } \\ \text { DPA (22:5n-6) } & \\ & \end{array}$






\section{Reference intervals of LC-PUFAs}

Reference intervals were determined for the 297 7-9 year old Zimbabwean children. Five children (1.7 \%) had EPA levels below the 2.5 percentile $(0.09 \% w t / w t)$, while $6(2.0 \%)$ had EPA levels above the 97.5 percentile $(0.37 \% w t / w t)$. Five children $(1.7 \%)$ had DPA levels below the 2.5 percentile $(0.53 \% w t / w t)$ and $6(2.0 \%)$ were above the 97.5 percentile $(1.15 \% w t / w t)$. Six children $(2.0 \%)$ had DHA levels below the 2.5 percentile
$(1.35 \% w t / w t)$ and $6(2.0 \%)$ were above the 97.5 percentile $(2.93 \% w t / w t)$. Six children $(2.0 \%)$ had ARA levels below the 2.5 percentile $(7.85 \% w t / w t)$ and 7 $(2.4 \%)$ were above the 97.5 percentile $(12.92 \% w t / w t)$. The LC-PUFA reference intervals with $90 \% \mathrm{CI}$ as determined from the results of the 7-9 year old Zimbabwean children are shown in Table 3.

\section{Discussion}

To our knowledge, this is the first study to report blood levels of omega-3 and omega-6 LC-PUFAs and to determine LC-PUFA reference intervals in 7-9 year old Zimbabwean children.

The levels for omega-3 LC-PUFAs (EPA, DPAn-3 and DHA) of the children in this study were strikingly low, while those of omega-6 LC-PUFA (ARA) were surprisingly high compared to the determined reference intervals and to the results obtained from a UK study on similar age groups and biomarker [42] (EPA $0.20 \mathrm{v} 0.56$, DPAn-3 0.81 v 1.03, DHA 2.15 v 1.9, ARA 10.56 v 8.17). Generally, these children had very low omega-3 PUFAs and very high saturated fats, monounsaturated and omega- 6 fatty acids. The essential omega- 3 fatty acid, $\alpha-$ linolenic acid, which is the precursor of the omega-3 LC-PUFAs (EPA, DPA and DHA), mainly found in seeds, nuts and some vegetable oils, was also low in the children under study (median level $0.38 \% \quad \% t / w t$ ) compared to results obtained from a study on similar age groups and biomarker [42]. The highest EPA value

Table 3 Reference Intervals of LC-PUFAs (\% wt/wt) in Zimbabwean children aged 7-9 years

\begin{tabular}{|c|c|c|c|}
\hline \multirow[t]{2}{*}{ LC-PUFAs (\% wt/wt) } & \multirow{2}{*}{$\begin{array}{l}\text { Median (IQR) } \\
(n=297)\end{array}$} & \multicolumn{2}{|c|}{ Reference Intervals 7-9 years old $n=297$} \\
\hline & & 2.5th Percentile $(90 \% \mathrm{Cl})$ & 97.5th Percentile (90\% Cl) \\
\hline \multicolumn{4}{|l|}{ Omega-3 fatty acids } \\
\hline ALA (18:3n-3) & $0.38(0.29-0.47)$ & $0.17(0.160 .20)$ & $0.73(0.670 .86)$ \\
\hline EPA $(20: 5 n-3)$ & $0.18(0.15-0.23)$ & $0.09(0.080 .10)$ & $0.37(0.330 .45)$ \\
\hline DPA (22:5n-3) & $0.79(0.70-0.89)$ & $0.53(0.490 .57)$ & $1.15(1.071 .26)$ \\
\hline $\mathrm{DHA}(22: 6 n-3)$ & $2.14(1.87-2.42)$ & $1.35(1.261 .46)$ & $2.93(2.863 .16)$ \\
\hline Total n-3 PUFA & $3.55(3.22-3.87)$ & $2.55(2.392 .64)$ & $4.64(4.455 .10)$ \\
\hline \multicolumn{4}{|l|}{ Omega- 6 fatty acids } \\
\hline ARA $(20: 4 n-6)$ & $10.62(9.77-11.38)$ & $7.85(6.868 .32)$ & $12.92(12.57$ 13.55) \\
\hline Total n-6 PUFA & $38.10(36.72-39.39)$ & 33.18 (31.72 33.91) & $41.74(41.14$ 42.41) \\
\hline \multicolumn{4}{|l|}{ Other fatty acids } \\
\hline Total saturated & $38.55(37.45-39.74)$ & $35.82(35.5136 .12)$ & 42. 71 (41.50 43.18) \\
\hline Total monounsaturated & $16.20(15.33-17.61)$ & 13.75 (13.37 14.07) & $21.39(20.23$ 22.04) \\
\hline Total DMA & $3.29(3.00-3.62)$ & $2.44(1.992 .62)$ & 4.15 (3.96 4.38) \\
\hline ARA: EPA & $57.47(44.72-72.24)$ & $26.51(21.58$ 28.26) & $110.83(104.50144 .63)$ \\
\hline$\%$ n-3LC-PUFA: Total LC-PUFA & 18.42 (17.10 19.92) & $14.54(14.06$ 14.98) & $24.56(23.0026 .34)$ \\
\hline Total n-3 LC-PUFA & $3.13(2.83-3.49)$ & 2.14 (1.98 2.28) & $4.29(4.034 .71)$ \\
\hline Total n-6 PUFA: Total n-3 PUFA ratio & $10.82(9.83-11.79)$ & $7.91(7.428 .48)$ & 14.48 (14.04 15.13) \\
\hline
\end{tabular}

Fatty Acids Acronyms: ALA "a-Linolenic acid", EPA "Eicosapentaenoic acid", DPA "Docosapentaenoic acid", ARA Arachidonic acid", DMA Dimethylacetal 
obtained in this study of $0.55 \% w t / w t$ was lower than the mean values obtained from a study on similar age groups and biomarker [42]. Results of the present study also demonstrated the lowest EPA value of $0.06 \% w t / w t$ reported in apparently healthy children compared to results obtained from a study on similar age groups and biomarker [42]. This might be a reflection of the different geographical backgrounds, diet and genetic make-up of the children in the different studies.

The low EPA and high ARA levels are of health concern because they lead to very high ARA: EPA ratios, which are pro-inflammatory, and to very high total omega-6 PUFA: total omega-3 PUFA ratios [30]. The high ratios observed in this study reflect possible imbalances in the dietary intake of omega- 6 and omega- 3 rich foods. The imbalances could be as a result of contemporary changes in human nutrition caused by increased consumption of diets rich in saturated fats (rich in beef), monounsaturated and omega- 6 fatty acids including the use of cooking oils, vegetable oils and bread spreads rich in omega- 6 PUFAs, accompanied by a decreased intake of omega-3 PUFA-rich foods [50]. Deficiencies in DHA exposes children between the ages of 7 and 9 to impaired brain development during the 7-9 year old "Brain Spurt" [44], possibly leading to compromised intellectual development, academic performance, low verbal learning ability, memory and learning difficulties [33, 34].

The LC-PUFA levels of all parameters except DHA were lower in the present study compared to the expected values from the University of Stirling Aquaculture laboratory [39] that used the same method of analysis and sample type (DBS) as the present study (EPA $0.20 \mathrm{v} 0.91$, DPA 0.81 v 2.47, DHA 2.15 v 2.47, ARA 10.56 v 13.88). The median EPA level in the present study was similar to that obtained by Mohammed et al. on pregnant Zimbabwean black women [36], indicating a general view of the dietary intake of foods poor in omega-3 LC-PUFAs and $\alpha$-linolenic acid in the population.

Our findings of no differences by gender in median LCPUFAs levels were in agreement with those of Glaser et al. on a paediatric population [45]. However, another study on a paediatric population reported a more pronounced low omega- 3 and omega-6 LC-PUFA status in girls than boys [42], while another study reported slightly higher omega-6 ARA in boys than in girls [46]. Yet another study, reported that sex hormones (testosterone and oestrogen) influence the enzymatic synthesis of LC-PUFAs, leading to gender related differences in LC-PUFA status with higher levels occurring in adult females [29]. The reason for the lack of gender differences in LC-PUFA levels observed in this study was perhaps due to the younger age of the participants.

The observed differences in median EPA and ARA: EPA ratio across and between the children's age groups is probably due to differences in dietary content. The 7 year old children had lower EPA and higher ARA values leading to high ARA: EPA ratio. The low EPA values observed in the children understudy are however constrained by the lower sample size in this particular age group; hence the results should be interpreted with caution. These findings were similar to UK study on similar age groups and biomarker [42] and also similar to the study with European children though with no age dependence for ARA [46]. An Italian study with differences in fatty acids by age groups concluded that the differences resulted either from lower intakes or the rates of utilization and resulting physiological requirements which are higher in younger age groups compared to older age groups [51].

The study also determined DBS LC-PUFA reference intervals for the apparently healthy 7-9 year old Zimbabwean children. However, these DBS LC-PUFA reference intervals cannot be generalised to the rest of the population since the LC-PUFA results were from children from a select group born to a cohort residing in a peri-urban setting, which did not include rural and urban children. The determined LC-PUFA reference intervals were not comparable to those of three other studies which determined LC-PUFA reference intervals perhaps due to methodological differences $[15,45,46]$.

Our results showed generally low values across the omega-3 LC-PUFA range. The levels of these LC-PUFAs could be improved by identifying and encouraging the intake of locally available omega-3 LC-PUFA rich foods. Supplementation with EPA and DHA omega-3 fish oils and algae based oils to balance ARA levels is recommended in the children since low omega-3 LC-PUFA levels are recognized confounders of general health. Limited intake of ARA-rich foods is also recommended if the desirable total omega-6 PUFA: total omega-3 PUFA ratio of 1-4:1 [6] is to be achieved. There is need for a public awareness campaign on food sources rich in omega-3 LC-PUFAs and the benefits of omega-3 LCPUFAs throughout life. We recommend further studies on children under the age of 5 years and inclusion of children from rural and urban Zimbabwe to ascertain their omega-3 LC-PUFA levels. Results from such studies could be used as the basis for establishing reference intervals that can be generalized to the whole Zimbabwean paediatric population, as well as the basis for food fortification and the implementation of omega-3 LC-PUFA supplementation policies.

The study has a number of limitations. Firstly no dietary intake assessment was done during specimen collection to ascertain the practices that could explain the low omega-3 LC-PUFA levels, hence, the causes of low omega-3 LC-PUFA levels are assumption based. Secondly, the determined reference intervals are limited to the children born to the specified cohort as a limited age group was used for this study. The study population 
was also restricted to children in a peri-urban setting that may not be truly reflective of the Zimbabwean population. The determined DBS reference intervals could also not be compared to those from other populations because of analytical method differences $[15,45,46]$. Lastly, the three age groups were unequal and this could distort the distribution of omega-3 LC-PUFAs findings by age.

\section{Conclusion}

Nevertheless, this is an important study that observed very low EPA levels and very high ARA: EPA and total omega-6 PUFA: total omega-3 PUFA ratios ever reported in apparently healthy children. The findings of this research could be the basis for future omega-3 LCPUFA intervention studies in Zimbabwe. The techniques learnt for LC-PUFA analysis could the basis of technology transfer to Zimbabwe.

\begin{abstract}
Abbreviations
$\%$ wt/wt, \% weight to weight; ALA, a-linolenic acid; ARA, arachidonic acid; $\mathrm{BHT}$, butylated hydroxytoluene; Cl, confidence intervals; CVD, cardiovascular diseases; DBS, dried blood spot; DGLA, dihomo-gamma-linolenic acid; DHA, docosahexaenoic acid; DPA, docosapentaenoic acid; EFA, essential fatty acids; EPA, eicosapentaenoic acid; FADS, fatty acid desaturase; FAME, fatty acid methyl ester; GLC, gas liquid chromatography; IQR, inter-quartile ranges; LA, linoleic acid; LC-PUFA, long chain polyunsaturated fatty acids; PMTCT, prevention of mother to child transmission; SPE, solid phase extraction; $\mathrm{TXA}_{2}$, thromboxanes; TXA , thromboxanes
\end{abstract}

\section{Acknowledgements}

This study was funded by the Letten Foundation (Oslo, Norway). We would like to thank the Letten Research Center for access to samples, the participants, their parents and legal guardians without whom this study would not have been possible. We would like to express our gratitude to Professor Bell of the Institute of Aquaculture University of Stirling Scotland UK, for training the first author in the analysis of the fatty acids, James Dick, Elizabeth MacKinlay and Irene Younger for hands on training and analysis of the samples.

\section{Funding}

This research was funded by the Letten Foundation (Oslo, Norway). The funders had no role in study design, data collection and analysis, decision to publish, or preparation of the manuscript.

\section{Availability of data and materials}

The data will not be made available in order to protect the participants identity.

\section{Authors' contributions}

GM Conception of the study, designed the study, coordinated the study, processed the sample, analyzed the samples, acquisition of data, drafting of the manuscript, interpretation of laboratory data, performed statistical analysis, data interpretation, manuscript review final manuscript write up. PK participated in study design and coordination, clinical examination of the children, sample collection and manuscript review. WT helped in statistical analysis review, interpretation of data and manuscript review. BS-P conception of the study, participated in study design and coordination and manuscript review. MM assisted in study conception, participated in study design and coordination, assisted in sample processing, helped to draft the manuscript and manuscript review. CM participated in study design, coordination, assisted to draft the manuscript and critical manuscript review. All authors read and approved the final manuscript.

\section{Authors' information}

Grace Mashavave (gracemashavave@yahoo.com)

MSc Clinical Biochemistry (UZ), Department of Chemical Pathology,

University of Zimbabwe College of Health Sciences.
Patience Kuona (patiekuona@gmail.com)

MMED Paediatrics (UZ), Department of Paediatrics and Child Health,

University of Zimbabwe College of Health Sciences.

Willard Tinago (wtinago@gmail.com)

PhD, Medical Statistics (University College Dublin, Ireland), Department of Community Medicine, University of Zimbabwe College of Health Sciences. Babill Stray-Pedersen (babill.stray-pedersen@medisin.uio.no)

PhD, Division of Women and Children, Rikshospitalet, Oslo University Hospital and Institute of Clinical Medicine, University of Oslo, Norway. Marshall Munjoma (marshall@uz-ucsf.co.zw)

PhD, Epidemiology and Diagnosis of Sexually Transmitted Infections (University of Oslo, Norway), Department of Obstetrics and Gynaecology, University of Zimbabwe College of Health Sciences.

Cuthbert Musarurwa (curtbertm@yahoo.com)

MSc Clinical Biochemistry (UZ), MSc Clinical Epidemiology (UZ), Department of Chemical Pathology, University of Zimbabwe College of Health Sciences.

\section{Competing interests}

The authors declare that they have no competing interests.

\section{Consent for publication}

Not applicable.

\section{Ethics approval and consent to participate}

The study protocol and consent forms were approved by the Joint Research Ethics Committee: (JREC/170/12), and the Medical Research Council of Zimbabwe: (MRCZ/B/359). The consent forms were also approved by the Norwegian Research Ethics Committee. Permission to ship participant samples abroad for laboratory analysis was granted by the Research Council of Zimbabwe. Written informed consent to participate in the study and for long term specimen storage and shipping was obtained from parents or legal guardians and written assent was also obtained from all the children.

\section{Author details}

${ }^{1}$ Department of Chemical Pathology, College of Health Sciences, University of Zimbabwe, PO BOX A178, Avondale, Harare, Zimbabwe. ${ }^{2}$ Department of Paediatrics and Child Health, College of Health Sciences, University of Zimbabwe, Harare, Zimbabwe. ${ }^{3}$ Department of Community Medicine, College of Health Sciences, University of Zimbabwe, Harare, Zimbabwe. ${ }^{4}$ Division of Women and Children, Oslo University Hospital and Institute of Clinical Medicine, University of Oslo, Oslo, Norway. ${ }^{5}$ Department of Obstetrics and Gynaecology, College of Health Sciences, University of Zimbabwe, Harare, Zimbabwe.

Received: 6 October 2015 Accepted: 20 July 2016

Published online: 05 August 2016

\section{References}

1. Mozurkewich E, Berman DR, Chilmigras J. Role of Omega-3 Fatty Acids in Maternal, Fetal, Infant and Child Wellbeing. Expert Rev Obstet Gynecol. 2010;5(1):125-38.

2. Schuchardt JP, Huss M, Stauss-Garbo M, Hahn A. Significance of long-chain polyunsaturated fatty acids (PUFAs) for the development and behaviour of children. Eur J Pediatr. 2010;169(2):149-64.

3. Richardson AJ, Burton JR, Sewell RP, Spreckelsen TF and Montgomery P. Docosahexaenoic Acid for Reading, Cognition and behavior in Children Aged 7-9 Years: A Randomized, Control Trial (the DOLAB Study). PLoS ONE 2012;7(9):e43909. doi: 10.1371.

4. Das UN. Essential fatty acids: biochemistry, physiology and pathology. Biotechnol J. 2006;1:420-39.

5. Bailey N. Immunonutrition: the role of long chain omega-3 fatty acids. The Nutrition Practitioner Spring 2010;11(1):24-26.

6. Patterson E, Wall R, Fitzgerald GF, Ross RP, Stanton C. Health Implications of High Dietary Omega-6 Polyunsaturated Fatty Acids. J Nutr Metab. 2012. doi:10.1155/2012/539426.

7. Poudyal H, Panchal SK, Diwan V, Brown L. Omega-3 fatty acids and metabolic syndrome: Effects and emerging mechanisms of action. Prog Lipid Res. 2011;50:372-87.

8. Gunveen K, Cameron-Smith D, Garg M, Sinclair AJ. Docosapentaenoic acid (22:5n-3): A review of its biological effects. Prog Lipid Res. 2011;50:28-34. 
9. Kidd PM. Omega-3 DHA and EPA for Cognition, Behaviour, and Mood: Functional Synergies with Cell Membrane, Phospholipids. Altern Med Rev. 2007;12(3):207-27.

10. Das UN. Arachidonic acid and lipoxin A4 as possible endogenous antidiabetic molecules. Prostaglandins Leukot Essent Fatty Acids. 2013;88:201-10

11. Engler MM, Engler MB, Malloy M, Chiu E, Besio D, Paul S, Stuehlinger M, et al. Docosahexaenoic acid restores endothelial function in children with hyperlipemia: results from the EARLY Study. Int J Clin Pharmacol Ther. 2004;42(12):672-9.

12. Norris JM, Yin X, Lamb MM, Barriga K, Sifert J, Hoffman M, et al. Omega-3 Polyunsaturated Fatty Acid intake and Islet Autoimmunity in Children at Increased Risk for Type 1 Diabetes. J Am Med Assoc. 2007;298(12):1420-8.

13. Forsyth JS, Willatts P, Agostoni C, Bissenden J, Casaer P, Boehm G. Long chain polyunsaturated fatty acid supplementation in infant formula and blood pressure in later childhood: follow up of a randomized controlled trial. Br Med J. 2003;953-doi:10.1136/bmj.326.7396.953-955.

14. Daak AA, Ghebremeskel K, Hassan Z, Attallah B, Azan B, Elbashir MI, Crawford M. Effect of omega-3 (n-3) fatty acid supplementation in patients with sickle cell anemia: randomized, double-blind, placebo-controlled trial. Am J Clin Nutr. 2013;97:37-44.

15. Vilaseca MA, Gomez-Lopez L, Lambruschini N, Gutiérrez A, García R, Meavilla $\mathrm{S}$, et al. Long-chain polyunsaturated fatty acid concentration in patients with inborn errors of metabolism. Nutr Hosp 2011;26(1):128-136.

16. Beblo S, Reinhardt H, Demmelmair H, Muntau AC, Koletzko B. Effect of Fish Oil Supplementation of Fatty Acid Status, Coordination and Fine Motor Skills in Children with Phenylketonuria. J Pediatr. 2007;150:479-84.

17. Janczyk W, Socha P, Lebenesztein D, Wierzbicka A, Mazur A, NeuhoffMurawska J, Matusik P. Omega-3 fatty acids treatment of non-alcoholic fatty liver disease: design and rationale of randomised controlled trial. BMC Pediatr. 2013;13:85.

18. Nobili V, Alisi A, Corte CD, Risé P, Galli C, Agostoni C, Bedogni G. Docosahexaenoic acid for the treatment of fatty liver: Randomised controlled trial in children. Nutr Metab Cardiovasc Dis. 2013;23(11):1066-70.

19. Belanger SA, Vanasse M, Spahis S, Sylvester MP, Lippe S, L'heureux F, Ghadirian $P$, Vanasse CM, Levy E. Omega-3 fatty acid treatment of children with attention-deficit hyperactivity disorder: a randomized, double-blind, placebo-controlled study. Paediatr Child Health. 2009;14:89-98.

20. Bell JG, Miller D, MacDonald DJ, MacKinlay EE, Dick JR, Cheseldine S, et al. The fatty acid compositions of erythrocyte and plasma polar lipids in children with autism, developmental delay or typically developing controls and the effect of fish oil intake. Br J Nutr. 2010;103:1160-7.

21. Biltagi MA, Baset AA, Bassiouny M, Attia M. Omega-3 fatty acids, vitamin C, and Zn supplementation in asthmatic children: A randomized selfcontrolled study. Acta Paediatr. 2009;98:737-42.

22. Chalasani N, Younossi Z, Lavine JE, Diehl AM, Brunt EM, Cusi K, Charlton M, Snayal AJ. The diagnosis and management of non-alcoholic fatty liver disease: Practice guideline but the American Association for the liver diseases, American College of Gastroenterology, and the American Gastroenterological Association. Hepatology. 2012;55(6):2005-23.

23. Hodge L, Salome CM, Hughes JM, Liu-Brennan D, Rimmer J, Allman M, Pang D, Armour C, Woolcock AJ. Effect of dietary intake of omega-3 and omega-6 fatty acids on severity of asthma in children. Eur Respir J. 1998;11:361-5.

24. Miller MR, Yin X, Seifert J, Clare-Salzler M, Eisenbarth GS, Rewers M, Norris JM. Erythrocyte membrane omega-3 fatty acid levels and omega-3 fatty acid intake are not associated with conversion to type 1diabetes in children with islet autoimmunity: the Diabetes Autoimmunity Study in the Young (DAISY). Paediatr Diabetes. 2011;12:669-75.

25. Mankad DA, Dupuis A, Smile S, Roberts W, Brian J, Lui T, Genore L, Zaghloul D, laboni A, Marcon PMA, Anagnostou E. A randomized, placebo controlled trial og omega-3 fatty acids in the treatment of young children with autism. Molecular Autism. 2015;6(18). doi:10.1186/s13229-015-0010-7

26. Gibson RA, Muhlhausler B, Makrides M. Conversion of Linolenic acid and alpha-linolenic acid to long chain polyunsaturated fatty acids, with focus on pregnancy, lactation and the first 2 years of life. Matern Child Nutr. 2011;Suppl 2:17-26.

27. Glaser C, Lattka E, Rzehak P, Steer C, Koletzko B. Genetic variation in polyunsaturated fatty acid metabolism and its potential relevance for human development and health. Matern Child Nutr. 2011;7 Suppl 2:27-40.
28. Simopoulos AP. The importance of the ratio of omega-6/omega-3 essential fatty acids. Biomed Pharmacother. 2002;56:365-79.

29. Decsi T, Kennedy K. Sex-specific differences in essential fatty acids metabolism. Am J Clin Nutr. 2011;94(Suppl):1914s-1919S.

30. Richardson AJ, Ross MA. Fatty acid metabolism in neurodevelopment disorder: a new perspective on associations between attention-deficit/ hyperactivity disorder, dyslexia, dyspraxia, and the autism spectrum. Prostaglandins Leukot Essent Fatty Acids. 2000;63:1-9.

31. Das UN. A defect in Delta6 and Delta5 desaturases may be a factor in the initiation and progression of insulin resistance, the metabolic syndrome and ischemic heart disease in South Asians. Lipids Health Dis. 2010;9:130.

32. Infante JP, Huszagh VA. Secondary carnitine deficiency and impaired docosahexaenoic (22:6n-3) acid synthesis: a common denominator in the pathophysiology of disease of oxidative phosphorylation and betaoxidation. FEBS Lett. 2000;468:1-5.

33. Dalton A, Wolmarans P, Witthuhn RC, van Stuijvenberg ME, Swanevelder SA, Smuts CM. A randomised control trial in school children showed improvement in cognitive function after consuming a bread spread, containing fish flour from a marine source. Prostaglandins Leukot Essent Fat Acids. 2009;80:143-9.

34. Tichelaar HY, Smuts CM, Kvalsvig JD, et al. Randomised study of cognitive effects of omega-3 fatty acid supplementation in undernourished rural school children. S Afr J Clin Nutr. 2000;13:100. abstr.

35. Baumgartner J, Smuts CM, Malan L, Kvalsvig J, van Stuijvenberg ME, Hurrell RF, Zimmermann MB. Effects of iron and n-3 fatty acid supplementation, alone and in combination, on cognition in school children: A Randomized double-blind, placebo-controlled intervention in South Africa. Am J Clin Nutr. 2012;96(6):1327-38. doi: 10.3945/ajcn.112.041004.

36. Mahomed K, Williams MA, King IB, Mudzamiri S, Woelk GB. Erythrocyte omega-3, omega- 6 and trans fatty acids in relation to risk of preeclampsia among women delivering at Harare Maternity Hospital, Zimbabwe. Physiol Res. 2007:56(1):37-50.

37. Bailey-Hall E, Nelson EB, Ryan AS. Validation of a rapid measure of blood PUFA levels in humans. Lipids. 2008:43(2):181-6.

38. Ichihara K, Waku K, Yamaguchi C, Saito K, Shibahara A, Miyatani S, Yamamoto K: A convenient method for determination of the C 20-22 PUFA composition of glycerolipids in blood and breast milk. Lipids 2002;37(5): 523-6

39. Bell JG, Mackinlay EE, Dick JR, Younger I, Lands B, Gilhooly T. Using a fingertip whole blood sample for rapid fatty acid measurement: method validation and correlation with erythrocyte polar lipid compositions in UK subjects. Br J Nutr. 2011;106:1408-15.

40. Marangoni F, Colombo C, Galli C. A method for the direct evaluation of the fatty acid status in a drop of blood from a fingertip in humans: applicability to nutritional and epidemiological studies. Anal Biochem. 2004;326:267-72.

41. Baylin A, Campos H. The use of fatty acid biomarkers to reflect dietary intake. Curr Opin Lipidol. 2006;17(1):22-7.

42. Montgomery P, Burton JR, Sewell RP, Spreckelsen TF, Richardson AJ. Low blood long chain omega-3 fatty acids in UK children are associated with poor cognitive performance and behavior: a cross-sectional analysis from the DOLAB study. PLoS One. 2013;8(6):e66697.

43. Taborda-Barats L, Potter PC. Socio-epidemiological Aspects of Respiratory Allergic Diseases in Southern Africa. World Allergy Organization J. 2012;5:1-8.

44. Thatcher RW. Maturation of the human frontal lobes. Physiological evidence for staging. Dev Neuropsychol. 1991;1:397-419.

45. Glaser C, Demmelmair H, Sausenthaler S, Herbarth O, Heinrich J, Koletzko B. Fatty acid composition serum glycophospholipids in children. J Pediatr. 2010;157:826-31.

46. Wolters MSH, Foraita R, Galli C, Risé P, Moreni LA, Molnár D, Russo P, Veidebaum T, Tornaritis M, Vyncke K, Eiben G, Lacoviello L, Ahrens W. Reference values of whole-blood fatty acids by age and sex from European children aged 3-8 years. Int J Obes. 2014;38:S86-98.

47. Kurewa NE, Munjoma MW, Chirenje ZM, Rusakaniko S, Hussain A, StrayPedersen B. Compliance and loss to follow up of HIV negative and positive mothers recruited from a PMTCT programme in Zimbabwe. Cent Afr J Med. 2007;53(5-8):25-30.

48. Lepage $\mathrm{G}$, Roy CC. Direct transesterification of all classes of lipids in a one-step reaction. J Lipid Res. 1986;27:114-20.

49. Geffré A, Concordet D, Braun J-P, Trumel C. Reference Value Advisor: a new freeware set of macroinstructions to calculate reference intervals with Microsoft Excel. Vet Clin Pathol. 2011;40(1):107-12. 
50. Molendi-Coste O, Legry V, Leclercq IA. Why and How Meet n-3 PUFA Dietary Recommendations? Gastroenterol Res Pract. 2011;2011:364040

51. Rise P, Tragni E, Ghezzi S, Agostoni C, Marangoni F, Poli A, et al. Different patterns characterize Omega- 6 and Omega-3 long chain polyunsaturated fatty acid levels in blood from Italian infants, children, adults and elderly. Prostaglandins Leukot Essent Fatty Acids. 2013;89:215-20.

Submit your next manuscript to BioMed Central and we will help you at every step:

- We accept pre-submission inquiries

- Our selector tool helps you to find the most relevant journal

- We provide round the clock customer support

- Convenient online submission

- Thorough peer review

- Inclusion in PubMed and all major indexing services

- Maximum visibility for your research

Submit your manuscript at www.biomedcentral.com/submit 